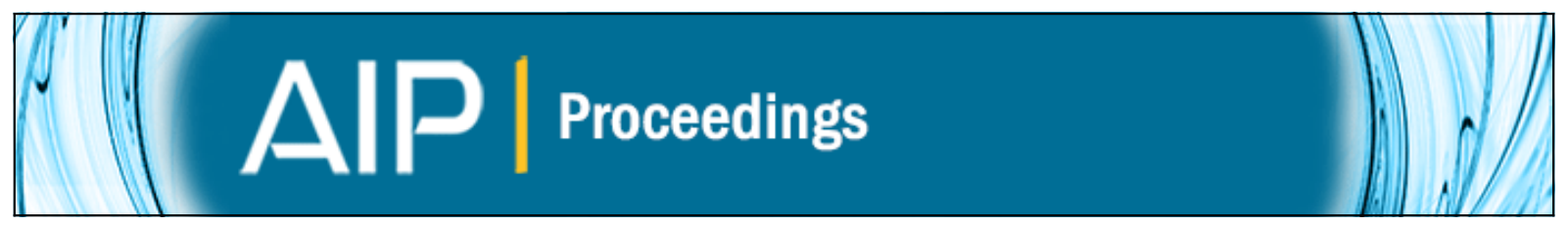

Challenges of simulating undrained tests using the constant volume method in DEM

Kevin J. Hanley, Xin Huang, Catherine O'Sullivan, and Fiona Kwok

Citation: AIP Conference Proceedings 1542, 277 (2013); doi: 10.1063/1.4811921

View online: http://dx.doi.org/10.1063/1.4811921

View Table of Contents: http://scitation.aip.org/content/aip/proceeding/aipcp/1542?ver=pdfcov

Published by the AIP Publishing

Articles you may be interested in

Hypocenter relocation using a fast grid search method and a 3-D seismic velocity model for the Sumatra region AIP Conf. Proc. 1554, 293 (2013); 10.1063/1.4820343

10,000 - A reason to study granular heat convection

AIP Conf. Proc. 1542, 38 (2013); 10.1063/1.4811864

Visualizing 3D Earthquake Simulation Data

Comput. Sci. Eng. 13, 52 (2011); 10.1109/MCSE.2010.120

Seismic Performance of a Base Isolated Structure by Shake Table Tests

AIP Conf. Proc. 1020, 1493 (2008); 10.1063/1.2963775

Recent Advances In Structural Vibration And Failure Mode Control In Mainland China: Theory, Experiments And Applications

AIP Conf. Proc. 1020, 137 (2008); 10.1063/1.2963760 


\title{
Challenges of Simulating Undrained Tests Using the Constant Volume Method in DEM
}

\author{
Kevin J. Hanley ${ }^{1}$, Xin Huang ${ }^{1,2}$, Catherine O'Sullivan ${ }^{1}$, Fiona Kwok ${ }^{2}$ \\ 1 Department of Civil and Environmental Engineering, Skempton Building, Imperial College London, London SW7 \\ $2 A Z$, United Kingdom \\ 2 Haking Wong Building, Department of Civil Engineering, The University of Hong Kong, Pokfulam Road, Hong \\ Kong
}

\begin{abstract}
Liquefaction during earthquakes can cause significant infrastructural damage and loss of life, motivating a fundamental study of undrained sand response using discrete element modeling (DEM). Two methods are widely used in DEM for simulating the undrained response of soil. One approach is to numerically couple the DEM code with a fluid model. Alternatively, if the soil is fully saturated and water is assumed to be incompressible, the volume of the sample can be held constant to simulate an undrained test. The latter has the advantage of being computationally straightforward, but the assumption of a constant volume can cause some issues which are discussed in this paper. Depending on the contact model selected, extremely high deviatoric stresses and pore water pressures can be generated for dense samples using the constant volume approach which are not observed in corresponding laboratory tests. Furthermore the results of these constant volume simulations tend to be sensitive to the strain rate selected. The evolution of particle size distribution caused by grain crushing is also ignored in most undrained simulations. For these reasons, authors often restrict the extent of the data presented to physically-realistic ranges and report results in nondimensional terms, e.g., using stress ratios (q/p') or stresses normalized by the initial confining pressure. This paper aims to highlight some of these issues, explore whether the constant volume approach is appropriate and make recommendations for future analysis of undrained soil behavior using DEM.
\end{abstract}

Keywords: Discrete element modeling, undrained simulations, pore water pressure, strain rate dependence. PACS: 45.50.Jf, 45.70.-n, 91.30.-f

\section{INTRODUCTION}

Undrained simulations are often used in discrete element modeling (DEM) studies of soil response. These simulations may be used to investigate phenomena such as liquefaction during earthquakes which can cause significant infrastructural damage and loss of life. Soil is often studied within a critical-state framework, and undrained simulations are very useful to supplement drained simulations when accurately finding the position of the critical state line. Two methods are widely used in DEM for simulating the undrained response of soil. One approach is to numerically couple the DEM code with a fluid model (e.g., using the coarse grid approach proposed by Tsuji et al., 1993 [1]). Alternatively, if the soil is fully saturated and water is assumed to be incompressible, the volume of the sample can be held constant to simulate an undrained test.

Although the latter is computationally straightforward, the assumption of a constant volume is not always appropriate and the simulation results are not physically realistic in all situations. Therefore it is crucial that researchers using the constant-volume approach to simulate undrained tests are aware of its limitations. The aim of this paper is to demonstrate some of the issues involved and make general recommendations on the use of the constant volume assumption in undrained DEM simulations of soil.

\section{MATERIALS AND METHODS}

The simulations presented in this paper were run using a version of the open-source code LAMMPS [2]. Some modifications were made to the base code to facilitate its use for undrained granular simulations. The most significant addition was a stress-control algorithm for periodically-bounded samples which included the ability to maintain the sample at a constant volume by adjusting the positions of lateral boundaries to compensate for axial shearing of the sample. This modified version of the code was successfully validated using the expressions developed by Thornton (1979) [3] for the stress ratios in a facecentered cubic assembly of uniform rigid spheres.

Two cubical samples containing 11,781 and 43,906 particles were created using a MATLAB script which successively placed each particle within a periodic space without contacting any preexisting particles. The particle size distributions (PSDs) of these samples are 
shown in Fig. 1, and were obtained by discretizing the PSD of a real Dunkirk sand. Only those particles with diameters greater than $250 \mu \mathrm{m}$ or $50 \mu \mathrm{m}(11,781$ or 43,906 particles, respectively) were included in these samples for computational tractability.

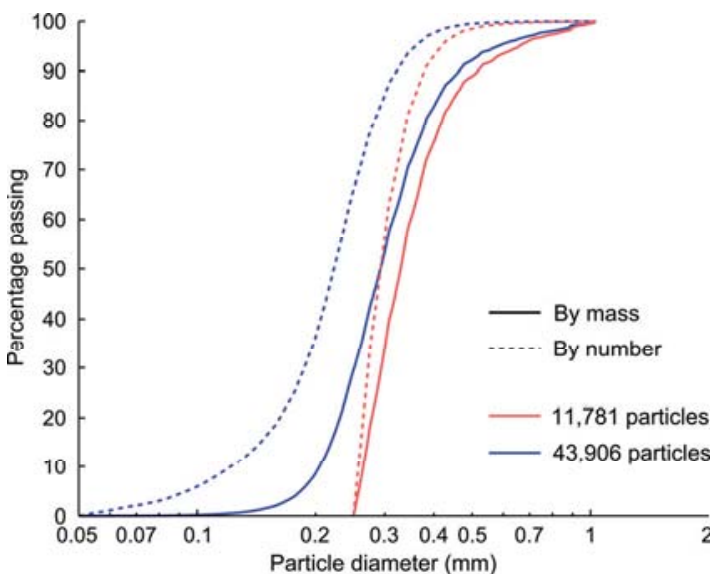

FIGURE 1. PSDs of the samples used in this study.

Once the radii had been generated, the particles were sorted in descending order and sequentially placed within the simulation domain using a random number generator. Two different contact models were allocated: a linear Hookean model and a simplified Hertz-Mindlin model. Each sample was isotropically compressed by moving the periodic boundaries closer together using a servo-control algorithm. Two large, dense samples were created using a friction coefficient of 0 during the preparation stage, while one smaller sample was created using a friction coefficient of 0.2 . All coefficients were increased to 0.5 once the target confining pressure, $\sigma_{\mathrm{o}}$, of $150 \mathrm{kPa}$ had been attained.

The shear modulus and Poisson's ratio were set at $29 \mathrm{GPa}$ and 0.2 , respectively, for the Hertzian contact model. The normal stiffness required for the linear model was calculated from the overlaps following isotropic compression of the Hertzian sample assuming that the samples had similar normal contact stiffnesses; the same value was used for the tangential Hookean stiffness for convenience. Those simulations using 43,906 spheres were run on 512 cores of HECToR (Phase 3 XE6), the principal HPC resource in the UK, with a timestep of 3 ns. Simulations using the smaller sample were run on a local cluster at Imperial College using a timestep of 10 ns. Gravity was not simulated. The void ratios of the prepared samples, defined as the volume of voids divided by the volume of particles, are given in Table 1. These are invariant throughout the subsequent triaxial simulations because of the constant volume assumption.
TABLE 1. Details of the prepared samples.

\begin{tabular}{cccc}
$\begin{array}{c}\text { No. of } \\
\text { Particles }\end{array}$ & $\begin{array}{c}\text { Contact } \\
\text { Model }\end{array}$ & $\begin{array}{c}\text { Friction Coeff. } \\
\text { During Prep. }\end{array}$ & $\begin{array}{c}\text { Void } \\
\text { Ratio }\end{array}$ \\
\hline 43,906 & Hookean & 0 & 0.4893 \\
43,906 & Hertzian & 0 & 0.4904 \\
11,781 & Hertzian & 0.2 & 0.6393 \\
\hline
\end{tabular}

All samples were subjected to undrained (i.e., constant volume) triaxial loading until an axial strain of $50 \%$ was attained. The smallest sample was tested using 11 different strain rates from $0.001 \mathrm{~s}^{-1}$ to $100 \mathrm{~s}^{-1}$. The simulations were carried out in a periodic cell and so shear bands did not form. In a real, physical test, shear band formation would reduce the pore water pressure.

\section{RESULTS AND DISCUSSION}

Fig. 2 shows the variation of deviator stress (q), mean effective stress (p') and excess pore water pressure $(\Delta u)$ with axial strain, where these quantities are defined by Eq. 1-3:

$$
\begin{gathered}
q=\sigma_{1}^{\prime}-\sigma_{3}^{\prime} \\
p^{\prime}=\frac{\sigma_{1}^{\prime}+\sigma_{2}^{\prime}+\sigma_{3}^{\prime}}{3} \\
\Delta u=\sigma_{o}-\sigma_{3}^{\prime}
\end{gathered}
$$

The stresses are extremely high at the end of the simulation for both contact models, but are an order of magnitude higher for the Hertzian then the Hookean model. Laboratory studies have shown that applying similar stresses to a real sand causes extensive particle crushing, even for strong particles, e.g., silica sands (Nakata et al., 2001 [4]). The omission of a suitable crushing model from any DEM simulation of a sand is questionable if similarly high stresses are attained.

A related problem which is specific to constantvolume undrained simulations of dense sands is the attainment of excess pore water pressures which have extremely large magnitudes, as shown on Fig. 2(b). Such large negative excess pore water pressures would require the use of a correspondingly large back pressure which may be unachievable using conventional laboratory apparatus. Furthermore, cavitation is likely to occur which was not captured by the simulations. McManus and Davis (1997) [5] found that cavitation of a dense silica sand occurred at a pore water pressure of around $-95 \mathrm{kPa}$, and thereafter the response changed from undrained to drained. It is possible that the concept of pore water pressure may not be meaningful if the pore fluid is not explicitly modeled. Furthermore Eq. 3, which was used to 
calculate $\Delta \mathrm{u}$, is based on the assumption that the soil skeleton is considerably more compressible than both

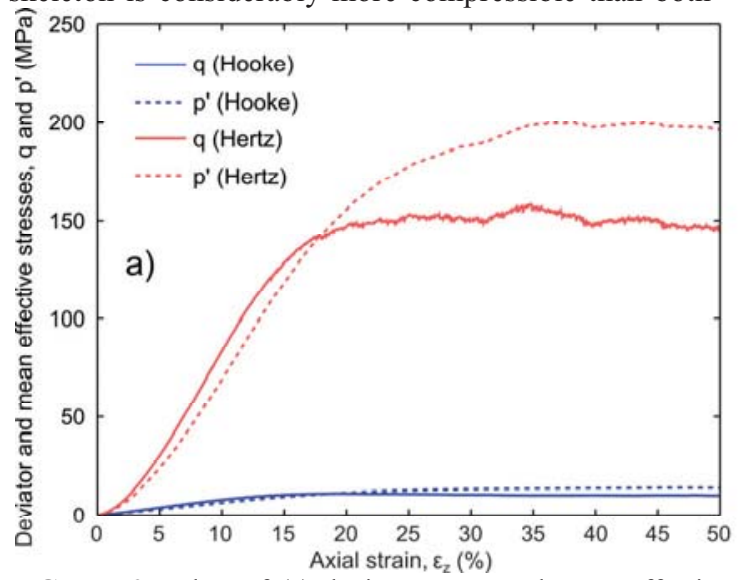

the particles and the pore fluid; in this case, neither assumption is valid.

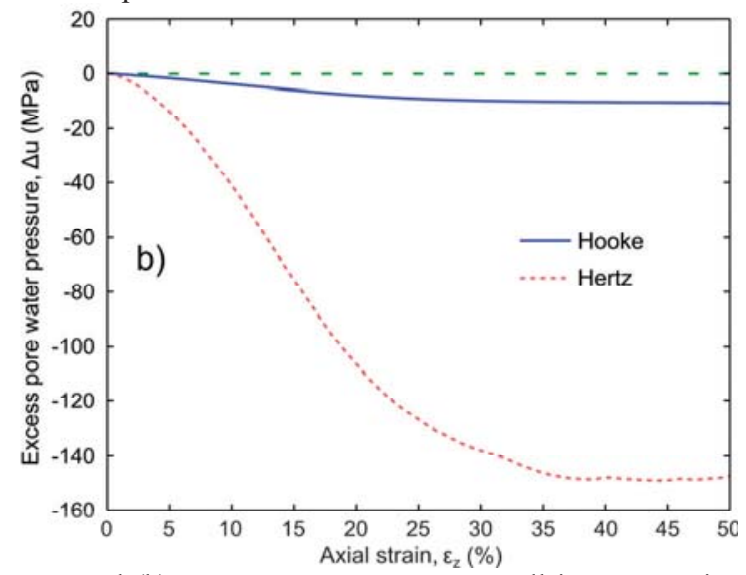

FIGURE 2. Plots of (a) deviator stress and mean effective stress, and (b) excess pore water pressure, all in MPa, against percentage axial strain, for triaxial tests conducted using samples containing 43,906 spheres and two different contact models.
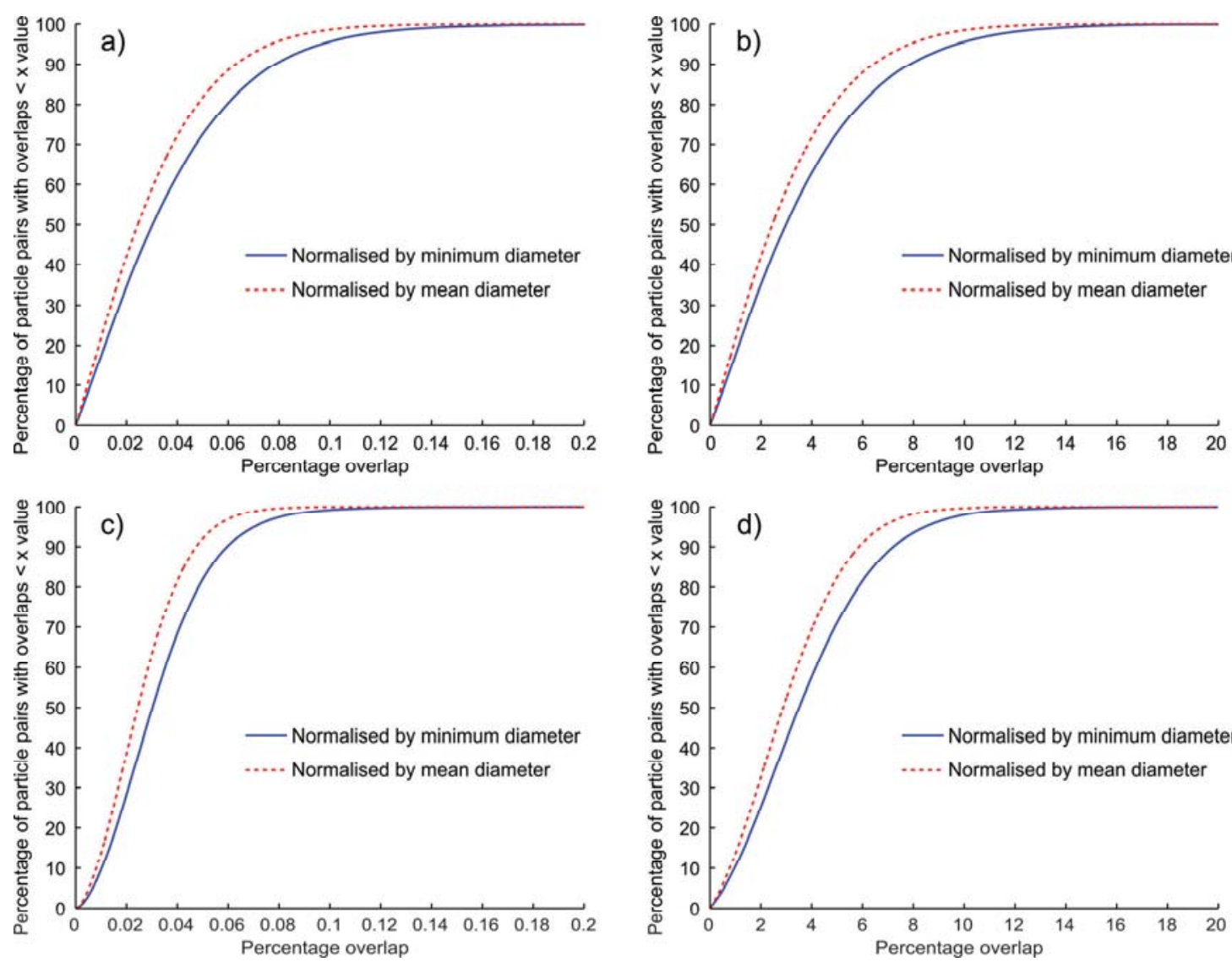

FIGURE 3. Percentages of particles with overlaps less than or equal to the $x$-axis value, normalized by both the minimum and mean diameters, for four cases: (a) sample with Hertzian contact model immediately after isotropic compression; (b) Hertzian model at 50\% axial strain; (c) Hookean model immediately after isotropic compression; (d) Hookean model at 50\% strain. 
The assumption of a constant volume becomes invalid when stresses reach the $\mathrm{MPa}$ range. The assumption is based on the incompressibility of water in a saturated sample. If the bulk modulus of water is taken to be $2.2 \mathrm{GPa}$, increasing the pressure to $10 \mathrm{MPa}$ causes a $0.5 \%$ decrease in water volume, while increasing it to $150 \mathrm{MPa}$ causes a decrease in volume of almost 7\%. Volume changes of this magnitude clearly invalidate the constant volume assumption.

In a soft-sphere DEM simulation, rigid particles are permitted to overlap at their contact points, equivalent to the deformation which occurs in reality. One of the fundamental assumptions that is made in DEM is that these overlaps are small relative to particle size so that point contact is approximated [6]. Although the threshold for the largest acceptable overlap is not well defined, a reasonable empirical guideline is to ensure that overlaps do not exceed $5 \%$. The overlaps are plotted in Fig. 3 for both simulations of 43,906 spheres. In these cumulative plots, the overlap between each pair of contacting particles is quantified as the sum of the particle radii minus the distance between the particle centers. Each overlap was normalized in two ways: by the smaller of the radii of the contacting particles or by the mean of the radii.

Fig. 3(a) and 3(c) shows that overlaps are very small immediately after isotropic compression to 150 $\mathrm{kPa}$ regardless of contact model. However overlaps are larger than $5 \%$ at almost $20 \%$ of contacts (normalized by mean diameter) by the end of the simulation, and close to $30 \%$ of contacts when normalized by the minimum diameter (Fig. 3(b) and 3(d)). The largest overlap exceeded $40 \%$ when normalized by the minimum diameter which is certainly too large.

\section{Strain Rate Sensitivity}

The issues discussed previously are specific to very dense samples, e.g., those created using low friction coefficients during sample preparation. Another issue which is seldom discussed in the literature is the effect of strain rate on the simulation results. Fig. 4 shows the effect of changing the strain rate by several orders of magnitude for the first $1 \%$ of axial strain (prior to liquefaction). The strain rate remains influential even when very low values are selected. Since loose materials are often of more interest to geotechnical engineers, this strain rate sensitivity may be more problematic than the generation of high stresses.

\section{CONCLUSIONS}

When very dense samples are used in a constant volume simulation, stresses may be generated which are extremely high regardless of the contact model selected. The magnitude of these stresses is sufficient to invalidate the constant volume assumption since water can no longer be considered to be incompressible. The large overlaps are also an indication that the DEM analyst should consider the simulations invalid. These observations demonstrate that clear criteria can be proposed to decide whether or not undrained simulations give valid data that is of interest to analysts trying to understand the behavior of real, physical systems.

Another issue which is important to consider is the strain-rate dependence of these constant-volume simulations. Since the results exhibit some sensitivity to strain rate, even at very low values, these tests should be run using the lowest feasible strain rate.

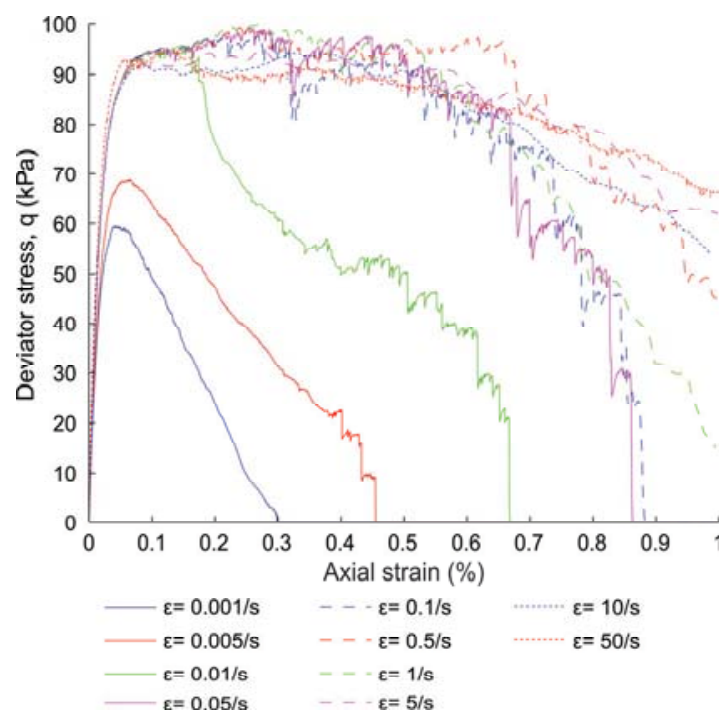

FIGURE 4. Effect of changing strain rate for a sample containing 11,781 spheres.

\section{ACKNOWLEDGMENTS}

Dr. Hanley would like to acknowledge funding from the Royal Commission for the Exhibition of 1851 .

\section{REFERENCES}

1. Y. Tsuji, T. Kawaguchi and T. Tanaka, Powder Technol. 77, 79-87 (1993).

2. S. Plimpton, J. Comput. Phys. 117, 1-19 (1995).

3. C. Thornton, Géotechnique 29, 441-459 (1979).

4. Y. Nakata, M. Hyodo, A. F. L. Hyde, Y. Kato and H. Murata, Soils Found. 41, 69-82 (2001).

5. K. J. McManus and R. O. Davis, Géotechnique 47, 173177 (1997)

6. D. O. Potyondy and P. A. Cundall, Int. J. Rock Mech Min. 41, 1329-1364 (2004). 